\title{
Mandenkan
}

MANDENIKAN Bulletin semestriel d'études linguistiques mandé

$52 \mid 2014$

Numéro 52

\section{Propositions pour l'orthographe du maninka (Guinée)}

Proposals for an orthography of Maninka (Guinea)

ПРЕДЛОЖЕНИЯ ПО СОВЕРШЕНСТВОВАНИЮ ОРФОГРАФИИ ЯЗЫКА МАНИНКА

ГВИНЕИ

Mamadi Diané et Valentin Vydrin

\section{OpenEdition}

Journals

\section{Édition électronique}

URL : https://journals.openedition.org/mandenkan/301

DOI : 10.4000/mandenkan.301

ISSN : 2104-371X

\section{Éditeur}

Llacan UMR 8135 CNRS/Inalco

\section{Édition imprimée}

Date de publication : 1 décembre 2014

Pagination : $3-21$

ISSN : 0752-5443

\section{Référence électronique}

Mamadi Diané et Valentin Vydrin, «Propositions pour l'orthographe du maninka (Guinée) », Mandenkan [En ligne], 52 | 2014, mis en ligne le 01 décembre 2014, consulté le 08 juillet 2021. URL : http:// journals.openedition.org/mandenkan/301 ; DOI : https://doi.org/10.4000/mandenkan.301

Ce document a été généré automatiquement le 8 juillet 2021.

Les contenus de Mandenkan sont mis à disposition selon les termes de la Licence Creative Commons Attribution - Pas d'Utilisation Commerciale - Partage dans les Mêmes Conditions 4.0 International. 


\title{
Propositions pour l'orthographe du maninka (Guinée)
}

\author{
Proposals for an orthography of Maninka (Guinea) \\ ПРЕДЛОЖЕНИЯ ПО СОВЕРШЕНСТВОВАНИЮ ОРФОГРАФИИ ЯЗЫКА МАНИНКА \\ ГВИНЕИ
}

Mamadi Diané et Valentin Vydrin

\section{NOTE DE L'AUTEUR}

Ce travail a bénéficié d'une aide de l'Etat gérée par l'Agence Nationale de la Recherche au titre du programme Investissements d'Avenir portant la référence ANR-10LABX-0083 (Labex EFL, Axe 6), et du projet de l'Université d'État de St. Petersbourg № 2.38.524.2013 « Langues d'Afrique subsaharienne: d'une analyse morphosyntaxique structurelle vers une synthèse fonctionnelle des éléments paradigmatiques de l'image linguistique du monde».

\section{Préambule}

1 Pendant les 25 ans écoulés depuis la promulgation de l'Ordonnance sur le nouvel alphabet des langues guinéennes (No. 019/PRG/SGG du 10 mars 1989) et la publication du guide d'orthographe (Le nouvel alphabet 1989), les recherches des linguistes guinéens et étrangers ont fait progresser considérablement la connaissance de la structure et $\mathrm{du}$ fonctionnement du maninka (Grégoire 1986; Creissels 2008 ; Vydrin \& Diané 2014, etc.).

D'autre part, beaucoup d'efforts ont été faits pour l'harmonisation de l'orthographe du mandingue au niveau international.

De nombreux phénomènes de la phonologie, de la morphologie et de la syntaxe du mandingue qui représentaient de grosses difficultés il y a un quart de siècle ont été 
clarifiés. Cela nous permet de présenter ici, sans aucune prétention à l'exhaustivité, des règles d'orthographe du maninka de Guinée qui tiennent compte des résultats des études faites par la communauté des linguistes mandéïsants et qui visent l'harmonisation, autant que possible, avec les systèmes d'orthographe élaborés pour les autres variétés du mandingue.

\section{L'alphabet}

4 L'alphabet de 1989 ne change pas. Il faut cependant préciser que dans la brochure de 1989 sur le nouvel alphabet, il y avait une confusion entre la notion de graphème (lettre) et phonème. Ainsi, les digraphes ( $a a, e e, \varepsilon \varepsilon$ etc. pour les voyelles longues et $g b$ ) étaient présentés en 1989 comme des «signes", alors qu'il s'agit en fait de combinaisons de signes. Cependant, selon la tradition établie, le digraphe $g b$ peut être considéré comme une unité alphabétique.

Compte tenu de cette rectification, l'alphabet maninka se présente comme suit : abcdecfggbhijklmnjo oprstuwy

\section{La phonologie et la correspondance entre les sons et les lettres}

\subsection{Les voyelles}

5 On distingue en maninka sept voyelles brèves :

$$
\begin{aligned}
& i-k i ́ l i \text { 'appeler', di 'donner' } \\
& e-s e ́ \text { 'arriver', kèle 'jaloux' } \\
& \varepsilon-d \bar{\varepsilon} \text { 'se réunir', té 'milieu' } \\
& a-d a ́ \text { 'bouche', kàla 'bâtonnet' } \\
& \text { o - kó 'dos', lò 's'arrêter' } \\
& o \text { - tòli 'pourrir', fodo 'champ' } \\
& u \text { - kú 'igname', sú 'nuit'. }
\end{aligned}
$$

\begin{tabular}{|c|c|c|c|c|c|c|}
\hline $\mathrm{i}$ & : ii & - & si & 'passer la nuit' : & : sìi & ‘s'asseoir' \\
\hline e & : ee & - & fére & 'fleur' & : fèere & 'vendre' \\
\hline$\varepsilon$ & $: \varepsilon \varepsilon$ & - & féf $\varepsilon$ & 'poivre africain' & : (kàba) f́́cfદe & 'omoplate' \\
\hline $\mathrm{a}$ & $:$ aa & - & fà & 'père' & fàa & 'tuer' \\
\hline o & : & - & kò & 'marigot' & : kòว & ‘sel’ \\
\hline 0 & $: 00$ & - & tó & 'rester' & : tóo & 'hutte, hangar' \\
\hline
\end{tabular}

$6 \quad n$ apparait comme une voyelle dans les pronoms $n+n^{\prime}$ 'moi', $n$ 'nous'. On peut dire que c'est une voyelle défective (à distribution restreinte).

7 Les sept voyelles longues sont désignées par des lettres doubles. Voici quelques exemples de paires minimales (ou quasi-minimales) qui ne se distinguent que par la longueur vocalique : 


\begin{tabular}{|l|l|l|l|l|l|l|l|}
\hline u & $:$ & uu & - & tù & 'gainer de cuir' & $:$ tùu & 'avant-bras'. \\
\hline
\end{tabular}

Le plus souvent, les voyelles longues en maninka standard (basé sur le dialecte maninkamori de Kankan) proviennent de la chute de la consonne vélaire $g$ entre deux voyelles identiques. Cette consonne est maintenue dans les dialectes de la zone de Siguiri, où on trouve les formes sìgi 's'asseoir', fàga 'tuer', tógo 'hutte', etc. On peut donc formuler une règle :

9 Là où on a deux voyelles identiques séparées par un $g$ dans le maninka de Siguiri, on a une voyelle longue dans le maninka standard.

10 Cependant, on trouve également des mots dont les voyelles longues sont d'origines différentes (soit elles étaient longues déjà dans la langue mère, soit il s'agit d'emprunts aux autres langues) : diina 'religion' (un emprunt à l'arabe), báa 'marque prédicative du conditionnel', mùume 'entier; du tout', siisaa 'asthme', etc.

11 Il faut faire très attention à la notation correcte de la longueur vocalique ; l'absence de distinction de la longueur est une faute grave car elle amène à la confusion.

\subsection{Les consonnes}

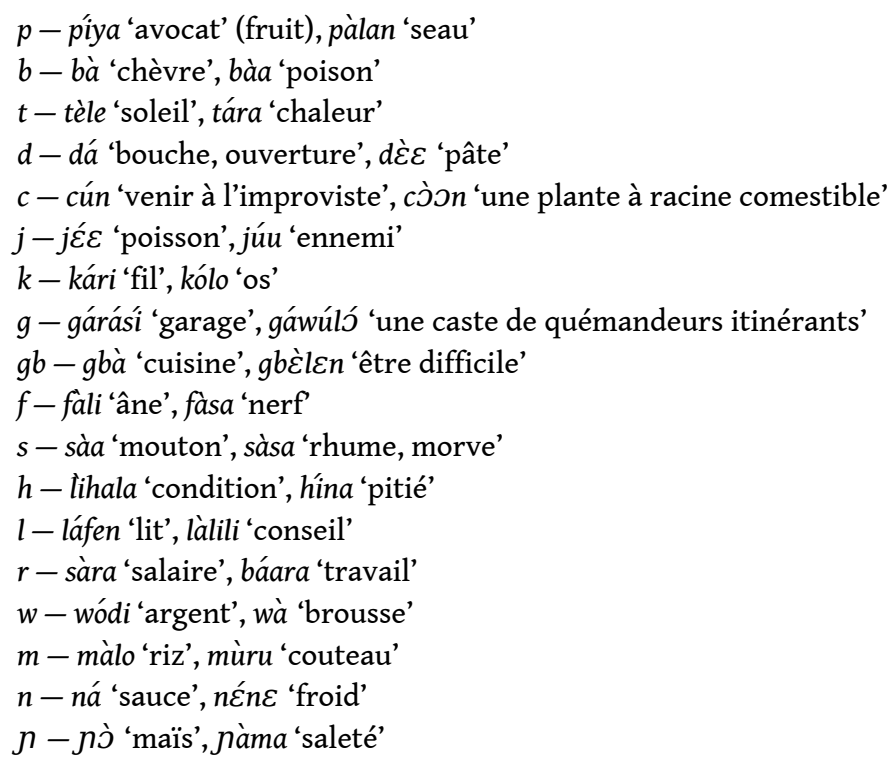

Quelques notes sur les consonnes.

1. $r$ apparaît presque uniquement à l'intérieur du mot.

Il y a deux types de $r$ en maninka : l'un est «stable ", il se présente toujours comme $r$ dans tous les dialectes centraux du maninka de Guinée. L'autre est en alternance libre avec $d$, et on trouve des variantes $t \grave{r} r \varepsilon$ vs $t \dot{\varepsilon} d \varepsilon$ 'marque du passé', sìri vs sìdi 'attacher', ró vs dó 'dans' (une postposition), kذ̀ro vs kذ̀do 'être vieux', etc. Ces variantes sont d'origine dialectale. En maninka de Kankan les deux variantes peuvent être prononcées même par un seul locuteur (pour certains mots, on peut préférer la variante avec un $r$, pour d'autres, celle avec un $d$ ). 
Pour les mots du deuxième type (à l'alternance $r \sim d$ ) il est suggéré de retenir la forme avec un $d$, pour deux raisons : d'abord, cela permet de distinguer entre les mots comme báda 'patrie' et bára 'place publique' (sinon, ces mots deviennent des homonymes : bára 'patrie' et bára 'place publique'). En outre, cela est plus conforme avec la pratique de l'écriture N'Ko, et il est souhaitable de ne pas créer des divergences inutiles entre les deux écritures maninka.

2. g est rare en maninka standard de Guinée, il n'apparaît que dans un nombre limité de mots, surtout des emprunts. Cependant, il serait erroné de considérer $g$ comme une variante du phonème $g b$; on trouve même quelques paires minimales (ou quasi-minimales) :

\begin{tabular}{|l|l|l|l|l|}
\hline gálan & 'un galant' & $:$ & gbálan & 'mirador' \\
\hline góro & 'gros type' & $:$ & gbóro & 'bouton (sur la peau)' \\
\hline gò & 'petite amie' & : & gbó & 'désagréable' \\
\hline
\end{tabular}

\subsection{Les structures syllabiques et la nasalisation}

Les types de syllabes en maninka sont les suivants ( $\mathrm{V}$ pour une voyelle, $\mathrm{C}$ pour une consonne) : $\mathrm{V}, \mathrm{CV}, \mathrm{CVn}^{1}$.

\subsection{1. Élément final $-n$}

Quand l'élément final - $n$ précède des suffixes, préfixes et mots auxiliaires ayant un $l, r$, ou $y$ au début, il les nasalise $: l \rightarrow n, r \rightarrow n, y \rightarrow n$. Il s'agit en particulier des morphèmes suivants :

yé/Jé (copule ; marque de l'habituel),

yé/Jé (marque prédicative du subjonctif),

-la/-na (suffixe de l'infinitif),

-la/-na (suffixe du nom de lieu),

-lan/-nan, rarement -ran (suffixe du nom d'instrument),

-li/-nin (suffixe du nom d'action),

$-y a /-n a$ (suffixe du nom d'état ou de qualité ; suffixe dérivatif de verbes qualitatifs),

$-l u /-n u$ (marque du pluriel),

lá: /ná (postposition locative à valeur générale),

ró/nó (postposition locative 'dans'; cette postposition apparaît également sous la

forme dó (sans alternance) qui est d'ailleurs préférable, suivant le principe sus-

mentionné),

la

lè $/ \grave{e}$ (particule de focalisation),

la:-/ná-(préfixe verbal à valeur causative),

ró-/nó- (préfixe verbal qui a également une variante dó- sans alternance, préférable

selon le principe sus-mentionné).

En contact avec la consonne nasalisée du suffixe ou préfixe suivant, l'élément nasal à la fin de la syllabe ne doit pas être omis. Par exemple : 


\begin{tabular}{|l|l|l|}
\hline Écriture correcte & Écriture incorrecte & Traduction \\
\hline A ye sòbo' dómunna. & *A ye sòbo' dómuna. & 'Il mange de la viande'. \\
\hline bàgbenna & *bàgbena & 'chevrier' \\
\hline
\end{tabular}

18 Les suffixes -ntan et -nte comportent un élément nasal qui ne doit pas être omis même si la racine du mot se termine aussi par un élément nasal. Dans ce dernier cas, une double nasale s'écrit: sènntan 'qui n'a pas de jambes'; kùnntan 'sans tête; inutile'; fisamannte 'le meilleur' (plutôt que *sèntan, *kùntan, *fisamante).

\subsubsection{Notation de la nasale finale suivie d'une consonne}

Suivi d'une consonne (surtout à l'intérieur d'un mot), l'élément nasal final de la syllabe s'adapte à cette consonne. Cependant, il est représenté à l'écrit invariablement par la lettre $n$ :

bànba 'crocodile' (prononcé [bàmbá]),

fánmajii 'modestie' (prononcé [fámmájii],

jànfa 'trahir' (prononcé [jàmfá]),

fanka 'force' (prononcé [fànká]),

jànjon 'exploit' (prononcé [jànjón])

kÉnde 'en bonne santé (prononcé [kéndé]).

\section{Les tons}

Selon la pratique établie dans l'écriture maninka en caractères latins, les tons ne sont pas notés. La même tendance prédomine dans tous les pays de l'aire manding. Suivant les recommandations des experts (B. Keita et al. 2003: 10), on propose de ne pas marquer systématiquement les tons dans les textes destinés aux locuteurs natifs (même si parfois cela peut amener à quelques confusions), sauf dans les publications pédagogiques. Cette pratique est motivée

- d'abord, par le fait que le contexte est le plus souvent suffisant pour désambiguïser le texte, «ce qui rend inutile les marques tonales qui apparaissent dès lors comme une surcharge dont on fait faire l'économie » (B. Keita et al. $2003: 10$ ) ;

- puis, par la difficulté d'apprendre la notation tonale par les néoalphabètes.

21 Le premier argument n'est justifié qu'en partie. On trouve en maninka des centaines des paires minimales qui ne se distinguent que par le ton, et le ton joue un rôle grammatical important. Il serait très facile de présenter des cas où le contexte ne permet pas la désambiguïsation ${ }^{2}$.

Le deuxième argument est dénié par l'expérience de l'enseignement du N'ko, écriture où les tons doivent être soigneusement notés. L'application d'une méthode d'enseignement élaborée par Solomana Kantè donne des résultats tout à fait satisfaisants, et les élèves des écoles N'ko maîtrisent parfaitement les tons maninka. On peut constater que la non-notation des tons maninka dans l'écriture latine est due plutôt à l'absence de méthode pédagogique appropriée et d'enseignants qualifiés. Sinon, l'orthographe de la langue française n'est pas plus facile que la notation tonale maninka, ce qui ne sert pas de prétexte à l'abrogation de son enseignement à l'école. 
Cependant, dans la situation actuelle, il serait idéaliste d'exiger une notation tonale obligatoire dans l'orthographe maninka en caractères latins; on risque de ne pas assurer le respect de cette demande par la grande majorité des utilisateurs de cette écriture. On proposera donc deux variantes d'orthographe, l'une sans notation tonale (pour le grand public), l'autre avec une telle notation (pour les publications linguistiques, dictionnaires, manuels de langue; il est recommandé cependant d'enseigner la notation tonale aux cours d'alphabétisation et, éventuellement, aux écoles).

\subsection{L'orthographe non-tonalisée}

24 L'orthographe non-tonalisée ignore les tons, à trois exceptions près : certains pronoms personnels, certaines marques prédicatives quasi-homonymiques, l'article tonal.

\subsubsection{Les pronoms personnels}

Il existe en maninka deux paires de pronoms personnels qui ne se distinguent que par le ton: 2PL álu (ou áyi) vs 3PL àlu (ou àyi), et 1SG ń vs 1PL ̀̀. Il est recommandé de noter

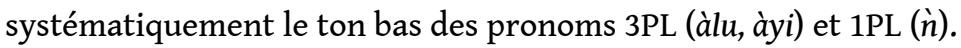

\subsubsection{Les marques prédicative}

Le maninka possède quatre marques prédicatives identiques en ce qui concerne leurs consonnes et voyelles, partiellement distinguées par leurs tons: kà (marque de l'infinitif), kà (marque de l'aoriste), ká (marque du prohibitif), ká (marque du verbe qualitatif). Il est recommandé de noter le ton bas sur les marques de l'infinitif et de l'aoriste, ce qui les distinguerait des deux autres marques.

$A$ kà sEnE' $k \varepsilon$ saron. 'Il a cultivé l'année dernière' (kà est la marque de l'aoriste).

Baba ka malo' sene de! 'Que Baba ne cultive pas le riz!' (ká est la marque du prohibitif).

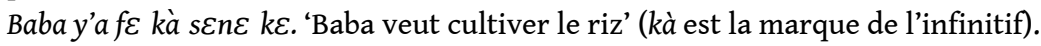

MalosenE' ka gbelen! 'Il n'est pas facile de cultiver le riz!' (ká est la marque du verbe qualitatif).

\subsubsection{L'article tonal}

L'article tonal se manifeste en maninka comme un ton flottant bas à la fin du mot. Il est recommandé de le désigner par l'apostrophe suivant le nom :

muso' kun' 'la tête de la femme' vs. musokun' 'une femme dynamique'.

\subsection{L'orthographe tonalisée}

Dans les textes tonalisés, il est recommandé de suivre les règles suivantes.

\subsubsection{Les diacritiques tonales}

Les diacritiques tonales utilisés sont l'accent aigu, á, pour le ton haut ; l'accent grave, $\grave{a}$, pour le ton bas ; le hachek, ă, pour le ton ascendant ; l'accent circonflexe, $\hat{a}$, pour un ton haut suivi d'un ton flottant bas (seulement dans quelques mots où ce ton flottant fait partie de la courbe tonale lexicale, comme jôn 'qui?', bî 'dizaine'). Le hachek est 
d'emploi très rare, seulement dans les mots de quelques classes tonales minoritaires, pour le ton ascendant précédant le ton haut. Le hachek n'est pas utilisé pour le ton ascendant précédant un ton bas: dans ce cas, on utilise l'accent grave, selon le principe : « le ton bas suivi d'un autre ton bas se réalise comme un ton ascendant ».

\subsubsection{Classes tonales majeures}

Pour les deux classes tonales majeures (Haut et Bas), les tons sont marqués sur les premières voyelles seulement, quelle que soit la longueur du mot :

bà 'chèvre, bá 'mère';

bàla 'porc-épique', bála 'balaphon'.

\subsubsection{Verbes à préfixes}


de ne l'appliquer qu'aux marques prédicatives, aux copules et aux conjonctions. On ne l'applique pas aux verbes:

Í d'à lón. 'Tu le sauras'.

Fántà n’à la sisce'. 'Fanta et son poulet',mais :

À dí à mà. 'Donne-le à lui'.

\subsection{Fusion de la copule ye} des formes géminées inhabituelles pour les locuteurs du maninka. On écrira donc :

Sáràn' ye bón' kJno (même si on prononcera: [Sáràǹn bón` kòno]). 'Saran (nom de femme) est dans la maison'.

Mùrú ye bón' kJno (même si on prononcera: [Mùrúù bón` kònò]). 'Le couteau est dans la maison'.

On trouve cependant des contextes où la copule peut être omise sans allonger la voyelle précédente ; il s'agit surtout de la position après la marque du passé $t \dot{d} d \varepsilon$. Dans ce cas, la copule peut ne pas être restituée à l'écrit :

Sóba' le tère yàn. 'Il y avait une grande ville ici' (l'écriture Sóba' le tère ye yàn étant également possible).

\section{La segmentation}

Les suffixes et les préfixes sont écrits collés, les mots auxiliaires sont écrits séparés.

\subsection{Mots auxiliaires en maninka}

Tableau 1. Marques prédicatives et copules

\begin{tabular}{|c|c|c|}
\hline Forme & Valeur & Exemple \\
\hline \multirow{2}{*}{ yé/jné } & copule & $\begin{array}{l}\text { Ń fà ye wà' do. 'Mon père est en brousse'. } \\
\text { Sáràn ne wà' do. 'Saran est en brousse'. }\end{array}$ \\
\hline & marque de l'habituel & $\begin{array}{l}\text { Tèle’ ye bóla. 'Le soleil brille'. } \\
\text { Fáràn ne kúmala féw. 'Faran parle vraiment!' }\end{array}$ \\
\hline$t \varepsilon^{\prime}$ & copule négative présentative & $\begin{array}{l}\text { Fàralafagban', jź } \varepsilon \text { té, sòbo té. 'Protoptère, ce } \\
\text { n'est pas un poisson, ce n'est pas un animal } \\
\text { de terre ferme'. }\end{array}$ \\
\hline bénà & futur prédictif & Háwà bénà jùmaya. 'Hawa sera belle’. \\
\hline dínà & futur prédictif & Mòri dinà sc̀nع' ké. ‘Mori cultivera le champ'. \\
\hline $\begin{array}{l}\text { ténà, } \\
\text { ténà }\end{array}$ & futur prédictif négatif & $\begin{array}{l}\text { Násù ténà gbà dònna. 'Nassou ne fera pas la } \\
\text { cuisine'. }\end{array}$ \\
\hline$d i$ & futur volitif ; habituel & Sáyon di wúya’ fó. ‘Sayon ment trop'. \\
\hline
\end{tabular}




\begin{tabular}{|c|c|c|}
\hline té, té & $\begin{array}{l}\text { futur volitif/habituel négatif; marque de } \\
\text { l'habituel négatif; copule négative } \\
\text { situative et équative }\end{array}$ & $\begin{array}{l}\text { Alimami' te dòls mìn. 'L'imam ne boit pas } \\
\text { d'alcool'. }\end{array}$ \\
\hline kà & aoriste & Bàba kà kúma. 'Baba a parlé’. \\
\hline báda & parfait & $\begin{array}{l}\text { Tàmati bada mò bórototo. 'La tomate est } \\
\text { devenu très mûre'. }\end{array}$ \\
\hline má & perfectif négatif & $\begin{array}{l}\text { Báara ma ké kúnùn. 'Il n’y a pas eu de travail } \\
\text { hier'. }\end{array}$ \\
\hline kà & infinitif & Ń ye à fè̀ kà sćbcli’ ké. 'Je veux écrire’. \\
\hline$k a ́$ & marque prédicative des verbes qualitatifs & $\begin{array}{l}\text { Ń na báara' ka gbèlen. 'Mon travail est } \\
\text { difficile'. }\end{array}$ \\
\hline mán & $\begin{array}{l}\text { marque prédicative négative des verbes } \\
\text { qualitatifs }^{3}\end{array}$ & $\begin{array}{l}\text { Ń na wùlu' man júu. 'Mon chien n'est pas } \\
\text { méchant'. }\end{array}$ \\
\hline yé/jé & subjonctif & $\begin{array}{l}\text { Ála ye î lákanda. 'Que Dieu te protège'. } \\
\text { Sáràn' ne ń tó ń súu' la. 'Que Saran me laisse } \\
\text { en paix'. }\end{array}$ \\
\hline kàná, ká & prohibitif (subjonctif négatif) & $\begin{array}{l}\text { Tólon kàná sc̀be sà. 'Que le jeu n'empêche pas } \\
\text { le sérieux'. }\end{array}$ \\
\hline báa & conditionnel & $\begin{array}{l}\text { Sàma' baa dòn, sán' di nà. 'Quand l'hivernage } \\
\text { arrive, il pleuvra'. }\end{array}$ \\
\hline
\end{tabular}

\subsection{Suffixes et préfixes en maninka}

Tableau 2. Les suffixes verbaux flexionnels

\begin{tabular}{|c|c|c|}
\hline Suffixe & Valeur & Exemple \\
\hline -bali & participe négatif & $\begin{array}{l}\text { Lón jànna á jànna, lón sébali té. 'Un jour est éloigné, mais il arrive } \\
\text { quand même' }\end{array}$ \\
\hline$-d a ́ /-\mathbf{r a ́}^{4}$ & $\begin{array}{l}\text { perfectif } \\
\text { intransitif }\end{array}$ & Bàba nàda kúnùn. 'Baba est venu hier'. \\
\hline$-l a /-n a$ & infinitif & $\begin{array}{l}\text { kàbi mòo séda nèc' ládanna... 'depuis que l'homme a su fabriquer le } \\
\text { métal...' }\end{array}$ \\
\hline $\begin{array}{l}-n i n /- \\
n \varepsilon n\end{array}$ & $\begin{array}{l}\text { participe } \\
\text { résultatif }\end{array}$ & à béc ládènen 'tout cela ensemble' \\
\hline$-\operatorname{san}$ & passé immédiat & Fàanin mèn sànsan ne nìn. 'Ce vêtement vient d'être acheté'. \\
\hline
\end{tabular}




\begin{tabular}{|l|l|l|}
\hline$-t a$ & $\begin{array}{l}\text { participe } \\
\text { potentiel }\end{array}$ & jió minta 'l'eau potable' \\
\hline$-t 0$ & $\begin{array}{l}\text { participe } \\
\text { progressif }\end{array}$ & $\begin{array}{l}\text { Wùlu' bòrito kába' le jé tèn. 'C'est à cause de la pierre que le chien } \\
\text { court'. }\end{array}$ \\
\hline
\end{tabular}

Tableau 3. Les préfixes verbaux

\begin{tabular}{|c|c|c|}
\hline Préfixe & Valeur & Exemple \\
\hline$l a a_{-}^{-}-/ n a^{-}$ & préfixe causatif & $\begin{array}{l}\text { Mu'ísà kà bà láminin. 'Moussa a abreuvé la chèvre'. } \\
\text { Háwà bada kòlonkalan' nála dùu' ma. 'Hawa a posé le } \\
\text { pilon par terre'. }\end{array}$ \\
\hline $\begin{array}{l}\text { dó- ró-/ } \\
\text { nó- }\end{array}$ & $\begin{array}{l}\text { préfixe désignant la localisation à } \\
\text { l'intérieur }\end{array}$ & Fódè bada kòlon’ dóbo. Fodé a curé le puits. \\
\hline$m a-$ & $\begin{array}{l}\text { préfixe désignant une action sur la } \\
\text { surface }\end{array}$ & Fántà bada bón' mámùuun. 'Fanta a peint la maison'. \\
\hline
\end{tabular}

Tableau 4. Les suffixes verbaux dérivationnels (verbe $\rightarrow$ nom)

\begin{tabular}{|c|c|c|}
\hline Suffixe & Valeur & Exemple \\
\hline$-b a a$ & $\begin{array}{l}\text { nom d'agent } \\
\text { occasionnel }\end{array}$ & $\begin{array}{l}\text { MòJ' kílibaa' ní î tà baa' té kélen dí. 'Celui qui appelle et } \\
\text { celui qui amène sont différents'. }\end{array}$ \\
\hline -baato & nom du subissant & $\begin{array}{l}\text { Sàbaato' kininkinin' ye à siijnoगnnu la. 'Les voisins ont pitié } \\
\text { du moribond'. }\end{array}$ \\
\hline$-1 a /-n a$ & $\begin{array}{l}\text { nom d'agent } \\
\text { permanent }\end{array}$ & $\begin{array}{l}\text { sébclila 'écrivain, celui qui écrit', sòsodamunna 'mangeur } \\
\text { de haricots' }\end{array}$ \\
\hline -li/-nin & nom d'action & sùsuli 'le pilage', sànnin 'un achat' \\
\hline $\begin{array}{l}\text {-lan/-nan, } \\
\text { rarement -ran }\end{array}$ & nom d'instrument & siilan 'chaise', tèzran 'hache' \\
\hline$-n t e$ & $\begin{array}{l}\text { nom d'agent } \\
\text { excessif }\end{array}$ & híncnte 'celui qui a pitié', jànfante 'traître' \\
\hline -baante & $\begin{array}{l}\text { nom d'agent } \\
\text { excessif }\end{array}$ & kálabaante 'personne maligne' \\
\hline
\end{tabular}

Tableau 5. Les suffixes nominaux

\begin{tabular}{|l|l|l|}
\hline Suffixe & Valeur & Exemple \\
\hline
\end{tabular}




\begin{tabular}{|c|c|c|}
\hline$-b a$ & augmentatif & $\begin{array}{l}\text { yíriba 'grand arbre' } \\
\text { wùlu júuba 'chien très méchant' }\end{array}$ \\
\hline- nin & diminutif & ko'snin 'petite affaire' \\
\hline$-k a$ & nom d'originaire/habitant du lieu & Kánkanka 'habitant/originaire de Kankan' \\
\hline$-l a /-n a$ & nom du lieu & $\begin{array}{l}\text { bíranna 'le village des beaux-parents', } \\
\text { núnkodola 'l'espace sous le nez' }\end{array}$ \\
\hline $\begin{array}{l}\text {-laka/- } \\
\text { naka }\end{array}$ & $\begin{array}{l}\text { nom de membre de famille ou habitant } \\
\text { du village; nom de membre d'une } \\
\text { classe }\end{array}$ & $\begin{array}{l}\text { Jàanclaka le yeń di ‘Je suis de la famille Diané'. } \\
\text { bílaka 'un chiffre de l'ordre des disaines' }\end{array}$ \\
\hline$-y a /-n a$ & suffixe de nom d'état ou de qualité & $\begin{array}{l}\text { mànsaya 'le statut du roi', fàntanja 'la position } \\
\text { du pauvre'; fádinja 'la férocité', }\end{array}$ \\
\hline
\end{tabular}

Tableau 6. Les suffixes adjectivaux

\begin{tabular}{|l|l|l|}
\hline Suffixe & Valeur & Exemple \\
\hline$-m a$ & $\begin{array}{l}\text { a) ornatif («pourvu de »); } \\
\text { b) suffixe des adjectifs dans } \\
\text { l'emploi prédicatif }\end{array}$ & $\begin{array}{l}\text { a) kálabuma 'courageux', hánkilima 'raisonnable, } \\
\text { intelligent' } \\
\text { b) Ń kà bòdo' jima' le tà 'J'ai pris le sac pendant qu'il } \\
\text { était mouillé'. }\end{array}$ \\
\hline$-n t a n$ & privatif & hánkilintan 'stupide', sènntan 'sans pieds, cul-de-jatte' \\
\hline$-m a n$ & $\begin{array}{l}\text { dérivation d'un adjectif à partir } \\
\text { d'un verbe qualitatif }\end{array}$ & forotofadinman 'piment chaud' \\
\hline$-t a$ & suffixe de focalisation d'adjectifs & $\begin{array}{l}\text { A báda foroto' fádinmanta sàn 'Il a acheté le piment le } \\
\text { plus chaud'. }\end{array}$ \\
\hline
\end{tabular}

Tableau 7. Le suffixe dérivationnel Verbe qualitatif $\rightarrow$ Verbe dynamique, Nom

\begin{tabular}{|l|l|}
\hline Suffixe & Exemples \\
\hline$-y a /-n a$ & fádinna 'devenir féroce ; la férocité', kòdoya 'vieillir ; vieillesse'. \\
\hline
\end{tabular}

Tableau 8. Le suffixe dérivationnel Numéral ordinal $\rightarrow$ Numéral cardinal

\begin{tabular}{|l|l|l|}
\hline Suffixe & Valeur & Exemples \\
\hline -nan & dérivation des numéraux ordinaux & sa'́: banan 'troisième', lóolunan 'cinquième' \\
\hline
\end{tabular}




\subsection{La marque du pluriel lú/nú}

La marque du pluriel lứ: $/ n u$, tout en étant un clitique (plutôt qu'un suffixe), s'écrit collée avec le mot précédent: sólu 'villages, villes', kùnnu 'têtes', sènjannu 'de longues jambes'.

\subsection{Les postpositions}

Les postpositions simples (dont les tons peuvent ne pas être marqués dans les textes) ${ }^{5}$ :

lá/ná (sens locatif général), dó/ró/nó 'dans', ḟ̀ 'près de', mà 'sur', kàn 'sur', bólo 'par', kódo 'sous', kóno 'à l'intérieur de', kùn 'dans la possession de', né 'devant', té 'entre', báda 'chez'.

\subsection{Les postpositions composées}

Les postpositions composées doivent être écrites en un seul mot, séparées des noms ou des pronoms qui les précèdent. On trouve en maninka les postpositions composées suivantes :

bólokódo 'sous la main de', dáfé 'à côté de', dákódo 'pour', dùulá 'en bas de', jùkódo

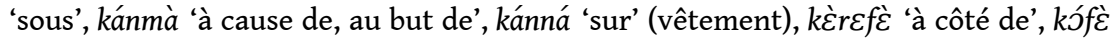
'derrière', kókàn 'à l'extérieur de', kókódo 'en soutien de', kómà 'derrière', kósòn, kùnná 'au-dessus de', jéfè 'devant', jámà 'devant', nána 'en présence de; celon', jákódo 'devant, sous les yeux', senfé̀ 'pendant', sènkódo 'en cachette de, à l'insu de', sèndó 'sous les pieds de' (sens figuré), sènná 'en marche', tćlá 'entre', tćmà 'autour de reins de', tòóf̀̀ 'à côté de', tòrof’è 'à côté de'.

\subsection{La marque possessive lá/ná}

La marque possessive la

à la nc̀cso' 'son vélo', mànsake' la sàmara' 'les chaussures du roi', àlu la bón' 'leur maison'.

En fait, par sa nature, elle peut être considérée comme une postposition.

\subsection{Les constructions nominales}

Les constructions nominales déterminatives Nom + Nom, qui sont tonalement compactes (possédant un seul contour tonal), s'écrivent collées : misisen 'patte de vache', mòofen 'une chose de quelqu'un, d'autrui'.

\section{7. Écriture des adjectifs}

\subsubsection{Les constructions nominales}

Les constructions nominales $\mathrm{N}+\operatorname{Adj}$ ( $\mathrm{du}$ type attributif) tonalement compactes s'écrivent collées :

sílajan' /síláján`/ 'une longue route', wòrofin' /wòròfin`/ 'le cola rouge', forotofadinman' / foròtòfádínmán / 'un piment fort'. 
Dans leurs emplois prédicatifs, les mêmes adjectifs (qui ne sont plus tonalement compacts avec les noms) s'écrivent séparément:

Sila' jàn. /sílá jàn/ 'La route est longue'.

Fòroto' fádinman. /foròtó' fádinmán/ 'Le piment est fort'.

\subsubsection{Les constructions attributives}

51 Les constructions attributives tonalement non-compactes s'écrivent séparement. Il s'agit des catégories suivantes des adjectifs:

- adjectifs dérivés de noms avec les suffixes -ma, -lama, -ntan ${ }^{6},-t 0$ :

- tò kòontan' 'le tô sans sauce', bólo jima' 'une main mouillée', súkara jillama' 'le sucre liquide', dén jito' 'un enfant peureux';

- adjectifs composés séparables :

- dénnin bólokJdomisen' 'un enfant qui touche à tout, enfant petit voleur' ;

- participes (résultatif, -nin/nEn; progressif, -to ; potentiel, -ta ; négatif, -bali) : mùso siinEn' 'une femme assise', dén sùnosto' 'un enfant endormi', màlo feereta' 'riz destiné pour la vente', nàmasa mòbali' 'banane non-mûre';

• adjectifs interrogatifs: bón jùman 'quelle maison ?', mùso jón' 'quelle femme ?'.

Pour les adjectifs (et participes) non-compacts, la différence entre leurs emplois attributif et prédicatif est exprimée par l'article tonal sur le nom précédent l'adjectif :

Tò' kذ̀ontan'. 'Le tô est sans sauce'.

Bólo' jima'. 'La main est mouillée'.

Súkara' jilama'. 'Le sucre est liquide'.

Dén' jito'. 'L'enfant est peureux'.

Dénnin' bólokJdo' miscnman'. 'L'enfant est un petit voleur'.

\subsection{Les numéraux}

Les numéraux (cardinaux comme ordinaux), ne formant pas de constructions tonalement compactes avec les noms, s'écrivent séparément :

mòo fila ‘deux personnes', lón kònondonan 'neuvième jour'.

De même pour le pronom-numéral joli :

Mùso joli?? 'Combien de femmes ?'

55 Les numéraux de deuxième ordre formés avec bi î s'écrivent collés (malgré l'absence de compacité tonale) ${ }^{7}$ :

bîlóolu 50, bîkònondo 90 .

\subsection{Les formes redoublées}

Les formes redoublées non-motivées (n'ayant pas de formes correspondantes nonredoublées) s'écrivent collées :

fidifidi 'balancer, tourner', búruburu 'séparer en petits morceaux'.

57 Les formes redoublées motivées (ayant des formes correspondantes redoublées) s'écrivent avec des traits d'union :

bòri-bòri 'courir dans tous les sens', tà-tà 'prendre à plusieurs reprises', jàma-jàma

'saletés (dispersées)'. 


\subsection{Les adverbes pré-verbaux} Selon les règles d'orthographe proposées ici, cette différence est exprimée par l'écriture collée/séparée et la présence/absence de l'article tonal (l'apostrophe).

- l'habituel: le verbe a un suffixe -la/-na (qui est tonalement compact avec le verbe et donc est écrit collé).

Dén' ye sùnojla bón' kJno. 'L'enfant dort dans la maison' (habituellement).

- le progressif (présent actuel): le verbe apparaît sous sa forme nominalisée, avec l'article tonal, suivie d'une postposition lá/ná (qui n'est pas tonalement compact avec le nom verbal précédent).

Dén' ye sùnoo' la bón' kJno. 'L'enfant dort dans la maison' (à ce moment).

Le verbe nominalisé peut former une construction tonalement compacte (et s'écrire en un seul mot) avec le nom exprimant le complément d'objet direct si ce nom est indéfini :

Mùsó' ye jísori' la bá' la. 'La femme est en train de puiser de l'eau dans le fleuve'.

Même un groupe nominal composé d'un nom + un modificateur (un adjectif, un nombre...) peut former une unité compacte avec le verbe nominalisé :

Mùsa' ye mùsofilafudu' la. 'Moussa est en train de se marier avec deux femmes'.

Si le nom est défini, il est tonalement autonome :

Mùsó' ye ji' sòri' la bá' la. 'La femme est en train de puiser l'eau dans le fleuve'.

\subsection{Les conglomérés}

Il s'agit des noms composés basés sur des constructions autres que celles admises dans les groupes nominaux. Ils gardent souvent les tons originaux de leurs composants, et dans ce cas les composants du congloméré sont écrits séparés :

siii-ń-féc 'étranger installé au village', súnkudun-č̀juu-gbàsi-bónsan 'un arbuste'.

Parfois, leurs tons sont uniformisés. Là où les tons originaux ne sont pas maintenus (et le congloméré porte un ton du type standard), on l'écrit collé :

siinfE 'le fait d'être étranger'.

\subsection{Quelques cas individuels}

Les séquences suivantes doivent être écrites collées :

dándo [dándó] 'quelques', dógbere [dógbéré] 'un autre', kàbini [kàbíní] 'depuis', kójùu [kójùu] ‘très’, kósebe [kósćbé, kósc̀bè] ‘très, beaucoup’, kónùman [kónùmàn] 
'bien, comme il faut', kókuda [kókúdá] 'de nouveau', mînké, mênké [mînké, mînkè,

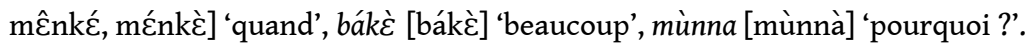

67 Les courbes tonales de ces séquences sont souvent différentes des tons de leurs composantes, et dans certains cas (comme mùnna) l'écriture collée permet leur différenciation des séquences homonymiques non-lexicalisées.

Par contre, les séquences suivantes, n'ayant pas perdu les courbes tonales de leurs composantes, s'écrivent séparées :

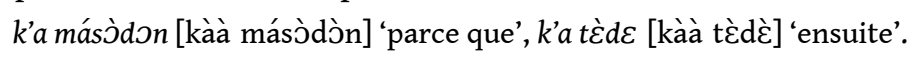

\section{BIBLIOGRAPHIE}

CREISSELS Denis, 2009, Le malinké de Kita, Köln, Rüdiger Köppe Verlag.

CREISSELS Denis, SAMBou Pierre, 2013, Le mandinka. Phonologie, grammaire, textes, Paris, Karthala. GRÉGOIRE Claire, 1986, Le maninka de Kankan. Éléments de description phonologique, Tervuren, Musée Royal de l'Afrique Centrale.

KEITA Boniface, TERA Kalilou, DIABY Moussa, et al., 2003, L'orthographe harmonisée du mandenkan (CASAS Monograph Series 24.) Cape Town, Centre for Advanced Studies of African Society.

Le nouvel alphabet des langues guinéennes, 1989, Conakry, Institut de recherches linguistiques appliquées.

VYDRIN Valentin, DIANÉ Mamadi, 2014, Segmentnaja fonologija gvinejskogo maninka (СЕГМЕНТНАЯ ФОНОЛОГИЯ ГВИНЕЙСКОГО МАНИНКА)[La phonologie ségmentale du maninka guinéen.] in A. Yu. ZHELTov (ed.). Antropologija i lingvistika. Materialy peterburgskikh ekspedicij v Afriku (АНТРОПОЛОГИЯ И ЛИНГВИСТИКА. МАТЕРИАЛЫ ПЕТЕРБУРГСКИХ эКСПЕДИЦИЙ В АФРИКУ) [L'anthropologie et la linguistique. Résultats des missions de terrain en Afrique par des chercheurs péterbourgois], St. Petersbourg, Musée d'Anthropologie et d'Ethnographie, pp. 128-144.

\section{ANNEXES}

\section{Échantillons de textes transcrits selon les règles d'orthographe qui précèdent}

\section{Un texte non-tonalisé}

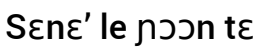

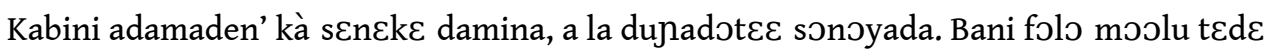

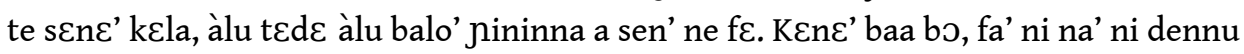

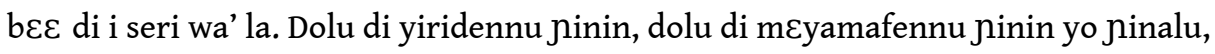
basalu, konolu, sobokunbalu yo minannu, siilu, sensennu, hali senbalu. 


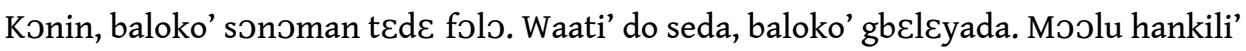

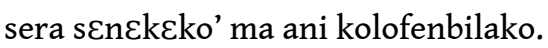

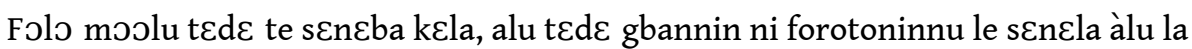
buudaninnu laminin' do. Kolofennu dando le $t \varepsilon d \varepsilon$ ye bilala àlu bolo : moว' $t \varepsilon d \varepsilon$ baa se àlu la buuda' la, i t $\varepsilon d \varepsilon$ di sisenin kelen-kelen yen, wulunin kelen, banen kelen,

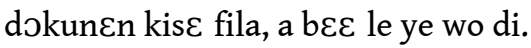

Mojlu tun man siya wo waati.

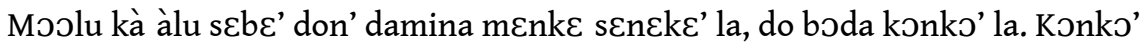
madooyada.

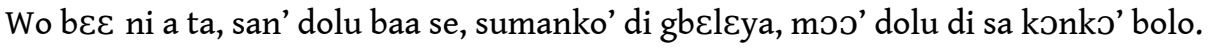

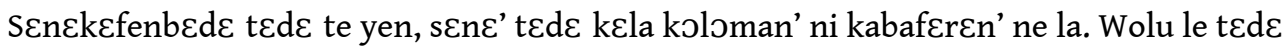

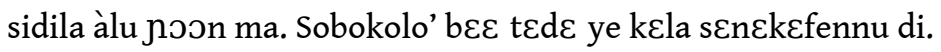

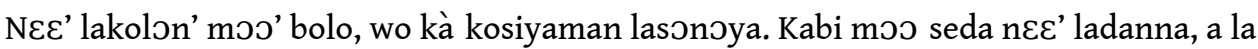

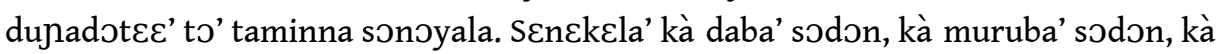
woroto' sodon, kà bine' ni tanba' sodon.

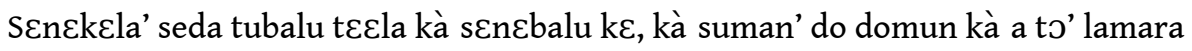

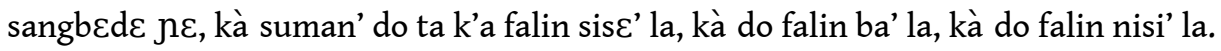

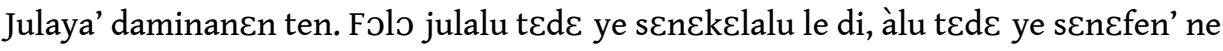

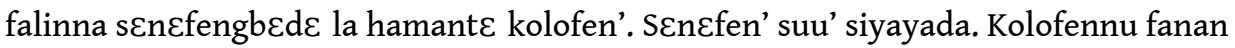
siyayada, dolu kà nisiwere' ladan, dolu kà saasulu' doben, dolu kà basulu' lo.

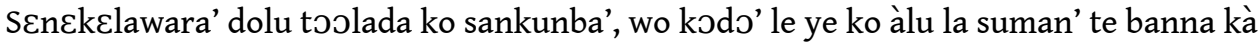
bo san' kun' do ma kà bila sangbed $\varepsilon$ kun' na.

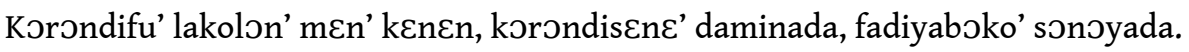
Faanin' toolada ko fadiyabo', ka fa' diyabo. Wo kodo le ye ko moว' baa fa, a di a miri faaninko' ma.

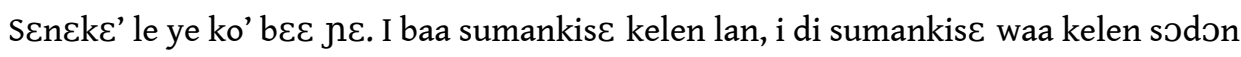

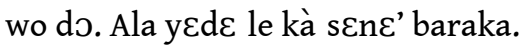

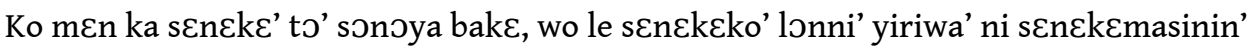

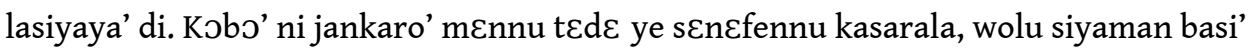

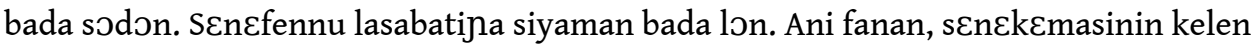

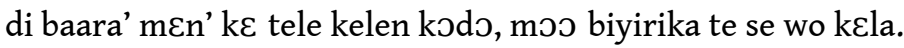

\section{Le même texte tonalisé}

\section{Sène' le nj̀on te}

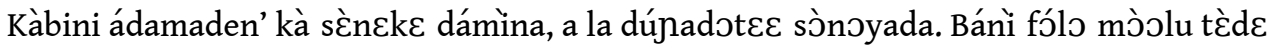

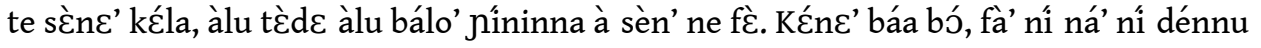
béc di î séri wà' la. Dólu di yíridennu nî́nin, dólu di mèyamafennu nî́nin yó nînalu, básalu, kònolu, sòbokunbalu yó mìnannu, sìilu, sénsennu, háli sènbalu.

Kònin, báloko' sònoman tèd $\varepsilon$ fólo. Wáati' dó séda, báloko' gbèleyada. Mòolu hánkili’

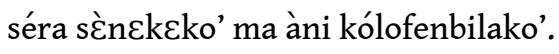




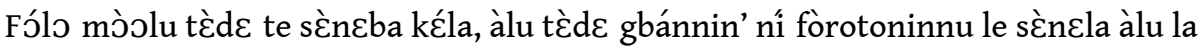
bùudaninnu láminin' do. Kólofennu dándo le tèd $\varepsilon$ ye bilala àlu bolo : mòo' tèd $\varepsilon$ báa sé àlu la bùuda' la, î tède di sìsenin kèlen-kelen yén, wùlunin kélen, bànen kélen, dòkunen kìse fila, à béc le ye wò di.

Mòolu tùn man síya wò wáati.

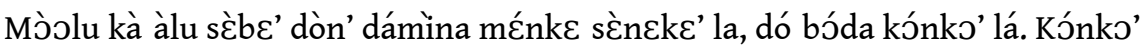
mádooyada.

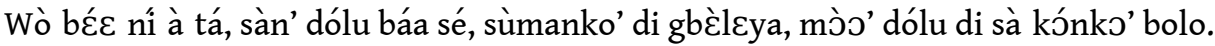

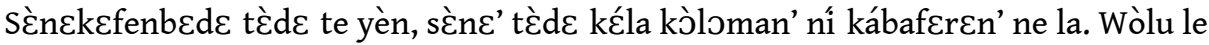

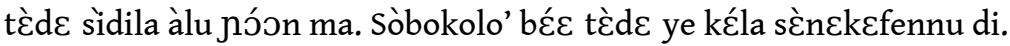

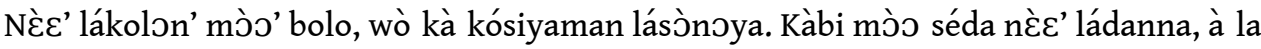

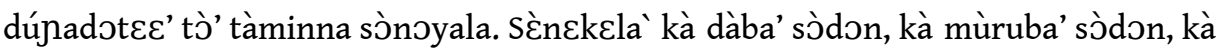
wòroto' sòdon, kà bìnع' ní tànba' sòdon.

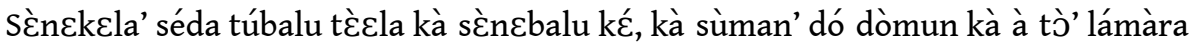
sàngbed $\varepsilon$ n $\varepsilon$, kà sùman' dó tà k'à fàlin sìse' la, kà dó fàlin bà' la, kà dó fàlin nìsi' la.

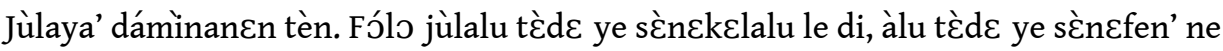

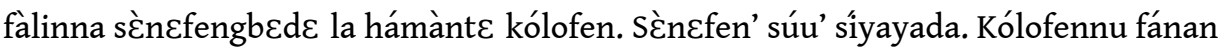
síyayada, dólu kà nìsiwere' ládan, dólu kà sàasulu' dóbèn, dólu kà bàsulu' lò.

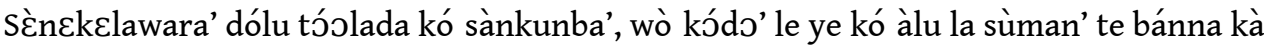
bó sàn' kùn' dó ma kà bila sàngbéde kùn' ná.

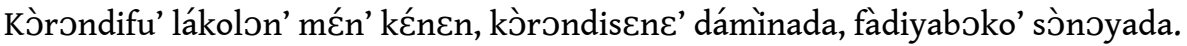
Fàanin' tóolada kó fádiyabo', kà fá' díyabo. Wò kódo` le ye kó mòo’ báa fá, à di à mìri fàaninko' ma.

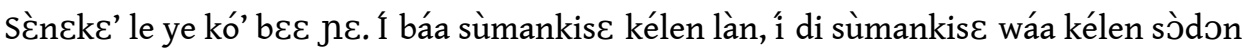
wò do. Ála yèd $\varepsilon$ le kà sène' bàraka.

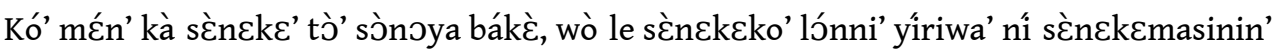
lásiyaya' di. Kóbo' ní jànkaro' ménnu tèd $\varepsilon$ ye sènعfennu kàsarala, wòlu síyaman bási'

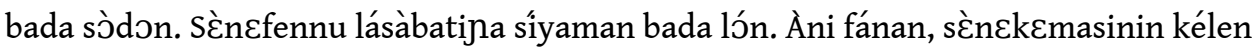
di báara’ mén` ké tèle kélen kodo, mòo bíyirika te sé wò kéla.

\section{NOTES}

1. Le type CVC est très marginal, il n'apparaît que dans quelques emprunts et adverbes expressifs.

2. On peut mentionner comme un cas anecdotique la devinette suivante : Jôn siinin ka jàn à lònin di? 'Qui est plus haut assis que débout ?' La bonne réponse estwùlu 'chien'. Écrit sans notation tonale, ce mot peut être compris commewúlu'penis'.

3. Un seul verbe qualitatif, jìn, s'utilise avec une variété de la marque négative à voyelle orale : à má jìn 'cela n'est pas bon'.

4. Ce morphème est tonalement autonome (il porte toujours un ton haut), ce qui peut être interprété comme un argument pour son statut de mot indépendant (d'ailleurs, en N'ko il est écrit séparé de la base verbale). D'autre part, la consonne $r \quad \sim d$ n'apparaît normalement pas dans la position initiale d'un mot, et en plus, aucun mot ne peut être inséré entre la base verbale et le morphème 
daí:-írá.

Nous proposons donc de traiter ce morphème comme un suffixe et de l'écrire collé à la base verbale.

Notons que les suffixes du perfectif intransitif

-táen mandinka (Creissels \& Sambou 2013 : 70-71) et -rá/-lá

en dioula véhiculaire de Côte d'Ivoire sont tonalement autonomes aussi, tandis que le suffixe

correspondant -ra/-la/-naen bambara ne l'est pas.

5. La plupart des postpositions sont très polysémiques; nous ne mentionnons ici qu'un sens par postposition. De même pour les postpositions composées ci-dessous.

6. L'adjectif kùnntan fait une exception : il est tonalement compact (et s'écrit collé) lorsque il exprime le sens 'inutile, sans valeur' : dénkunntan' 'un enfant vaurien'. Il n'est pas compacte lorsque il a son sens propre 'sans tête' : wùlu kùnntan' 'un chien sans tête'.

7.

Cette option a été choisie par la grande majorité des participants de la réunion des chercheurs de

l'Institut de Recherches Linguistiques Appliquées (Conakry) en janvier 2014. Elle contredit la pratique des autres langues manding (cf. l'article sur l'orthographe du bambara dans ce numéro), mais s'accorde avec la pratique du N'ko.

\section{RÉSUMÉS}

Les auteurs proposent dans cet article des règles d'orthographe du maninka de Guinée en écriture latine. Ils avancent des solutions à certains problèmes discutables: l'écriture de l'élément nasal final de la syllabe suivie d'une consonne ; l'inventaire des consonnes; des règles de notation tonale sont présentées en deux variantes, complète et allégée. Une attention spéciale est faite aux contractions et à la segmentation; des listes complètes des morphèmes et des mots auxiliaires sont données.

The authors advance orthography rules for the Maninka of Guinea in Roman script. They suggest solutions to many problems under discussion for many years: spelling of the syllable-final nasal element followed by a consonant; the inventory of the consonants, and some others. Two variants of rules for tone marking, a "light" and a "full" one, are formulated. Special attention is paid to contraction and segmentation. Exhaustive lists of auxiliary morphemes and words are given.

АВТОРЫ ФОРМУЛИРУЮТ ПРАВИЛА ОРФОГРАФИИ ДЛЯ ГВИНЕЙСКОГО МАНИНКА (В ЛАТИНСКОЙ ГРАФИКЕ). ПРЕДЛАГАЕТСЯ РЕШЕНИЕ НЕКОТОРЫХ ПРОБЛЕМ, ОСТАВАВШИХСЯ ДИСКУССИОННЫМИ В ТЕЧЕНИЕ ПОСЛЕДНИХ ДЕСЯТИЛЕТИЙ, ТАКИХ КАК ОБОЗНАЧЕНИЕ КОНЕЧНОСЛОГОВОГО НОСОВОГО ЭЛЕМЕНТА В ПОЗИЦИИ ПЕРЕД СОГЛАСНЫМ; ИНВЕНТАРЬ СОГЛАСНЫХ ФОНЕМ, И НЕКОТОРЫХ ДРУГИХ. РАЗРАБОТАНЫ ДВА ВАРИАНТА ТОНАЛЬНОЙ НОТАЦИИ - ОБЛЕГЧЁННЫЙ И ПОЛНЫЙ. ОСОБОЕ ВНИМАНИЕ УДЕЛЕНО ПРАВОПИСАНИЮ СТЯЖЁННЫХ ФОРМ И СЕГМЕНТАЦИИ ЗВУКОВЫХ ПОСЛЕДОВАТЕЛЬНОСТЕЙ. ПРИВОДЯТСЯ ПОЛНЫЕ СПИСКИ СЛУЖЕБНЫХ МОРФЕМ И СЛОВ. 
INDEX

Thèmes : maninka, langues mandé

Keywords : Maninka Language, Mande Languages, Orthography, Tone Marking motsclesru ЯЗЫК МАНИНКА, ЯЗЫКИ МАНДЕ, ОРФОГРАФИЯ, ТОНОВАЯ НОТАЦИЯ Mots-clés : orthographe, notation tonale

\section{AUTEURS}

MAMADI DIANÉ

IRLA, Conakry

langues2014nationales@gmail.com

\section{VALENTIN VYDRIN}

LLACAN, INALCO, USPC, Paris

Université d'État de St. Petersbourg

vydrine@gmail.com 